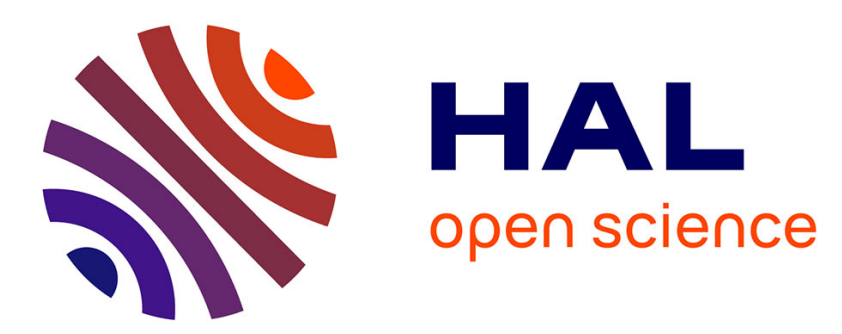

\title{
Models and simulations of dynamic frictional contact of a thermoelastic beam
}

\author{
Kenneth L. Kuttler, Yves Renard, Meir Shillor
}

\section{To cite this version:}

Kenneth L. Kuttler, Yves Renard, Meir Shillor. Models and simulations of dynamic frictional contact of a thermoelastic beam. Computer Methods in Applied Mechanics and Engineering, 1999, 177, pp.259-272. 10.1016/S0045-7825(98)00384-3 . hal-00690540

\section{HAL Id: hal-00690540 \\ https://hal.science/hal-00690540}

Submitted on 26 Apr 2018

HAL is a multi-disciplinary open access archive for the deposit and dissemination of scientific research documents, whether they are published or not. The documents may come from teaching and research institutions in France or abroad, or from public or private research centers.
L'archive ouverte pluridisciplinaire HAL, est destinée au dépôt et à la diffusion de documents scientifiques de niveau recherche, publiés ou non, émanant des établissements d'enseignement et de recherche français ou étrangers, des laboratoires publics ou privés. 


\title{
Models and simulations of dynamic frictional contact of a beam
}

\author{
K.L. Kuttler ${ }^{\mathrm{a}}$, Y. Renard ${ }^{\mathrm{b}}$, M. Shillor ${ }^{\mathrm{c}, *}$ \\ ${ }^{2}$ Department of Mathematical Sciences, Michigan Technological Universitv, Houghton, MI 49931. USA \\ "Laboratoire de Modelisation et Calcul, Université J. Fourier de Grenoble, 3804I Grenoble cedex. France \\ 'Department of Mathematics and Statistics, Oakland University, Rochester, MI 48309, USA
}

We investigate a mathematical model for the dynamic thermomechanical behavior of a viscoelastic beam that is in frictional contact with a rigid moving surface. Friction is modeled by a version of Coulomb's law with slip dependent coefficient of friction, taking into account the frictional heat generation. We prove the existence and uniqueness of the weak solution, describe an algorithm for the numerical solutions and present results of numerical simulations, including the frequency distribution of the noise generated by the stick/slip motion. We also show that when the surface moves too fast there are no steady solutions and therefore the system is thermally unstable.

\section{Introduction}

We investigate the dynamic behavior of a one-dimensional thermomechanical system which consists of a beam that is in frictional contact with a rigid obstacle. Our interest lies in the well-posedness of the model and in the stick-slip behavior of the contacting end when the coefficient of friction depends on the relative slip velocity. There is considerable interest in dynamic contact with friction, both mathematically and in applications, especially, since the noise and vibration characteristics of engineering systems are recognized as possible obstacles in product marketing.

We consider a 'simple' setting, so that we can concentrate on the behavior that the solutions exhibit, without having to deal with technical mathematical difficulties. Indeed, the purpose is to gain insight into the possible types of behavior of systems in frictional contact, and use this insight in two- and three-dimensional problems which are much more complicated, both mathematically and numerically.

Often, in engineering literature, the coefficient of friction is assumed to jump from a static value to a smaller dynamic value (see e.g. [8] and the survey [11] and references therein). Models with a variable coefficient of friction are recent in the mathematical literature (see e.g. [1,5,7,9] and references therein). Here, we assume that the coefficient of friction is slip dependent, but the dependence is continuous.

The isothermal problem of frictional contact between a beam and a rigid foundation can be found in [2] where a number of different contact conditions were investigated, and the existence of weak solutions established. In addition, we consider here the thermal aspects of the process. We model the contact with a version of Coulomb's law which was derived recently in [10] with a slip dependent friction coefficient. The considerable heat generated by frictional contact is taken into account. Indeed, it is found that if too much heat is generated, the problem does not have a steady solution, which indicates thermal instability (see e.g. $[3,12]$ and references therein).

* Corresponding author. 
The classical model is presented in Section 2. It consists of the beam equation for a viscoelastic material and the heat equation. The problem is nonlinear and coupled because of the contact and frictional heat generation boundary conditions. Since, in general, there are no classical solutions to problems with contact conditions, we present a weak formulation in Section 3. We state our existence and uniqueness result in Theorem 3.1. The proof is based on a sequence of solutions to a family of linearized and regularized problems. The existence of unique weak solutions to these problems follows from an abstract result of Kuttler [6], which has been generalized and improved in [7]. Then, we obtain a priori estimates on these solutions and pass to the limit when the regularization parameter tends to zero. In Section 4 we describe a Crank-Nicholson type of algorithm for the thermoelastic problem. Then, we present four different results of numerical simulations. These are: (i) constant coefficient of friction $\mu$ and constant foundation velocity $v_{*}$; (ii) constant $\mu$ and oscillatory $v_{*}$; (iii) slip dependent $\mu$ and oscillatory $v_{*}$; and (iv) temperature dependent $\mu$ and oscillatory $v_{*}$. The last case is not covered by our theory. For each of the simulations, we present the Fourier transform of the contacting end's velocity which shows the possible noise characteristics of the solution. In Section 6 we obtain the steady solution of the problem when the frictional heat generation is not too large, since then there is no steady state and the system is thermally unstable. We summarize our findings in Section 7. The results are very interesting and indicate that the problem warrants a more thorough investigation.

\section{The model}

In this section, we present the model for the process. The physical setting, depicted in Fig. 1, is as follows. A beam is rigidly attached at its left end; its right end is in frictional contact with a moving surface. The area-center of gravity of the beam in its (stress free and isothermal) reference configuration coincides with the interval $0 \leqslant x \leqslant 1$. For $T>0$, we set $\Omega_{T}=(0,1) \times(0, T) ; u=u(x, t)$ and $\theta=\theta(x, t)$, defined on $\Omega_{T}$, represent the vertical displacements and the temperature of the beam, respectively.

We employ the Kelvin-Voigt law of viscoelasticity for the shear stress

$$
-\sigma=-\sigma(x, t)=c^{2} u_{x+x}(x, t)+d c^{2} u_{t x u x}(x, t),
$$

where $c$ is proportional to the elastic modulus, $d(d \geqslant 0)$, is the viscosity coefficient, and subscripts denote partial derivatives. When $d=0$ the material is simply elastic. Let $f$ denote the density (per unit length) of vertical applied forces. The equation of motion, in dimensionless form, takes the form $u_{t}-\sigma_{x}=f$, and therefore,

$$
u_{\| \prime}+c^{2} u_{u \ldots x}+d c^{2} u_{t \ldots \ldots x}=f \quad \text { in } \Omega_{T}
$$

The energy equation can be written, in dimensionless form, as

$$
\theta_{t}-k \theta_{s, x}=0 \text { in } \Omega_{T},
$$

where $k$ is the (scaled) coefficient of thermal diffusivity.

The initial conditions take the form

$$
u(x, 0)=u_{0}(x), \quad u_{t}(x, 0)=v_{0}(x), \quad \theta(x, 0)=\theta_{0}(x),
$$

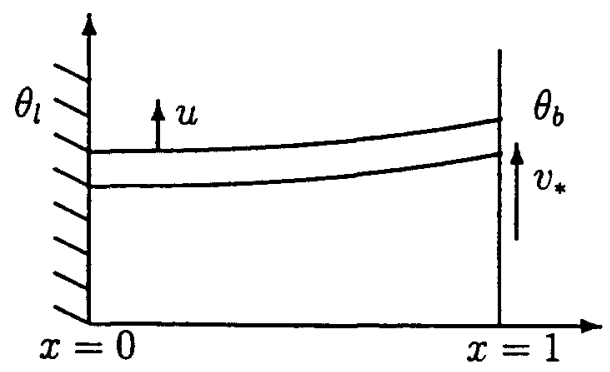

Fig. 1. The deflected beam in frictional contact. 
for $0 \leqslant x \leqslant 1$. Here $u_{0}, v_{0}$, and $\theta_{0}$ represent the beam's initial displacement, velocity and temperature, respectively.

The beam is rigidly attached to the left wall, and has the wall's temperature, denoted by $\theta_{l}=\theta_{l}(t)$, thus

$$
u(0, t)=0, \quad u_{x}(0, t)=0, \quad \theta(0, t)=\theta_{t}(t),
$$

where $0 \leqslant t \leqslant T$. We allow for time dependence of $\theta_{l}$ in case it is periodic, although below we simply set it to be the constant ambient temperature.

Our main interest lies in the frictional contact process at the right end. The surface is assumed to move with vertical velocity $v_{*}=v_{*}(t)$, which may be time dependent. A simple argument shows that the contact pressure that results from the horizontal thermal expansion of the beam is given by

$$
p(t)=\left(\alpha \int_{0}^{1} \theta(x, t) \mathrm{d} x\right)_{+},
$$

where $f_{+}=\max \{f, 0\}$, and $\alpha$ is proportional to the coefficient of thermal expansion. We use $(\cdot)_{+}$to make sure that when the beam shrinks and loses contact because of cooling the pressure vanishes.

Let $\mu$ denote the coefficient of friction, then $\mu h$ is the friction bound, where $h$ is given by

$$
h=h(t)=p(t)(1-\beta p(t))_{+} .
$$

Here, $\beta$ is a very small positive constant related to the wear and hardness of the surface. This version of the friction bound has been derived in [10]. When $\beta=0$ we obtain the usual Columb's law.

We are interested, in particular, in the case when the coefficient of friction depends on the relative slip between the end and the moving surface, thus

$$
\mu=\mu\left(\left|u_{t}(1, t)-v_{*}\right|\right) .
$$

This is often the case in applications, and for recent results and further references see e.g. $[1,5,7,9]$.

The modified Coulomb's friction condition is

$$
\begin{aligned}
& |\sigma(1, t)| \leqslant \mu h(t), \\
& |\sigma(1, t)|=\mu h(t) \text { then } u_{t}(1, t)-v_{*}(t)=-\lambda \sigma(1, t), \\
& |\sigma(1, t)|<\mu h(t) \text { then } u_{t}(1, t)=v_{*}(t) .
\end{aligned}
$$

When the shear stress $\sigma$ equals $\pm \mu h$ the end is in a slip motion, and the shear direction is opposite to the slip (here $\lambda \geqslant 0$ is an unknown multiplier); when $|\sigma|<\mu h$ the end sticks to the obstacle and moves with it, which is called stick motion.

The sum of the moments acting on the free end is assumed zero. The thermal boundary condition is chosen as

$$
k \theta_{s}(1, t)=\mu h(t)\left|u_{t}(1, t)-v_{*}(t)\right|-h_{b}\left(\theta(1, t)-\theta_{b}(t)\right) .
$$

The first term on the right-hand side represents the frictional heat generated during slip motion and is proportional to the pressure and the slip; the second term represents the thermal interaction between the end and the surface which has temperature $\theta_{b}=\theta_{b}(t)$.

We summarize the classical problem as: Find a pair $\{u, \theta\}$ such that

$$
\begin{array}{ll}
u_{t \prime}+c^{2}\left(u_{x x x x}+d u_{t x x x x}\right)=f & \text { in } \Omega_{T}, \\
\theta_{t}-k \theta_{x x}=0 & \text { in } \Omega_{T}, \\
u(x, 0)=u_{0}(x), \quad u_{t}(x, 0)=v_{0}(x), \quad 0 \leqslant x \leqslant 1, & \\
\theta(x, 0)=\theta_{0}(x), \quad 0 \leqslant x \leqslant 1, & \\
u(0, t)=0, \quad u_{x}(0, t)=0 \\
\theta(0, t)=\theta_{t}, \\
u_{x x}(1, t)+d u_{t x x}(1, t)=0, \\
k \theta_{x}(1, t)=\mu h(t)\left|u_{t}(1, t)-v_{*}(t)\right|-h_{b}\left(\theta(1, t)-\theta_{l}(t)\right),
\end{array}
$$




$$
\begin{aligned}
& |\sigma(1, t)| \leqslant \mu h(t), \\
& |\sigma(1, t)|=\mu h(t) \text { then } \quad u_{t}(1, t)-v_{*}(t)=-\lambda \sigma(1, t), \\
& |\sigma(1, t)|<\mu h(t) \quad \text { then } \quad u_{t}(1, t)=v_{*}(t)
\end{aligned}
$$

for $0 \leqslant t \leqslant T$, where $h$ is given by (2.6) and $p$ by (2.5).

Note that the coupling between the mechanical and thermal parts is through the boundary conditions (2.16) and (2.17) which introduce nonlinearities into the problem.

We assume that $\mu=\mu(r)$ is a smooth bounded function that depends on the magnitude of the slip $r=\left|u_{i}-v_{*}\right|$. It satisfies $\mu(0)=\mu_{0}>0$, is decreasing and tends asymptotically to $\mu_{x}>0$. For the sake of convenience, we assume that

$r \mu^{\prime}(r)$ is bounded,

so that $\nu(r) \equiv r \mu(r)$ is Lipschitz continuous. The case when $\mu$ is a graph with a vertical segment at the origin is more complicated and will be considered in the sequel.

The friction condition precludes, in general, the existence of smooth solutions to the problem, therefore, we turn to a weak formulation for it.

\section{Existence of the weak solution}

We consider a weak abstract formulation of problem (2.9)-(2.17) and prove that it has a unique weak solution. Without loss of generality we assume $\theta_{l}=0$. We define

$$
V \equiv\left\{u \in H^{2}(0,1): u(0)=u_{x}(0)=0\right\},
$$

$H \equiv L^{2}(0,1), \mathscr{V} \equiv L^{2}(0, T ; V)$ and $\mathscr{H} \equiv L^{2}(0, T ; H)$, and let $\gamma: V \rightarrow \mathbb{R}$ denote the trace on the right boundary of $[0,1]$ given by $\gamma u=u(1)$. Next, we define the operator $A: V \rightarrow V^{\prime}$ by

$$
\langle A u, v\rangle \equiv \int_{0}^{1} u_{x,} v_{x x} \mathrm{~d} x
$$

Moreover, let $E \equiv\left\{\theta \in H^{1}(0,1): \theta(0)=0\right\}, \mathscr{E} \equiv L^{2}(0, T ; E)$, and define the operators $B, N(t, \cdot): E \rightarrow E^{\prime}$ by

$$
\langle B \theta, \eta\rangle \equiv \int_{0}^{1} \theta_{x} \eta_{x} \mathrm{~d} x, \quad\langle N(t, \theta), \eta\rangle \equiv-h_{b}\left(\gamma \theta-\theta_{b}(t)\right) \gamma \eta,
$$

where, with a slight abuse of notation $\gamma$ is also the trace of $E$ onto the right endpoint, i.e. $\gamma \theta=\theta(1)$. For $\theta \in \mathscr{H}$ and $v \in \mathscr{V}$ we define $g(\theta, \gamma v) \in \mathscr{E}^{\prime}=L^{2}\left(0, T ; E^{\prime}\right)$ by

$$
\langle g(\theta, \gamma v)(t), \eta\rangle \equiv \mu\left(\left|\gamma v(t)-v_{*}(t)\right|\right)\left|\gamma u(t)-v_{*}(t)\right| h(\theta)(t) \gamma \eta
$$

where

$$
h(\theta) \equiv \alpha\left(\int_{0}^{1} \theta \mathrm{d} x\right)_{+}\left(1-\beta \alpha \int_{0}^{1} \theta \mathrm{d} x\right)_{+},
$$

for $\theta \in H$. It is straightforward to show that when $\beta>0$.

LEMMA 3.1. There exists a positive constant $K$ such that $|h(\theta)| \leqslant K$, and

$$
\begin{aligned}
& |h(\theta)-h(\eta)| \leqslant K|\theta-\eta|_{H}, \\
& \left|\mu\left(r_{1}\right) r_{1}-\mu\left(r_{2}\right) r_{2}\right| \leqslant K\left|r_{1}-r_{2}\right| .
\end{aligned}
$$

Next, we introduce $\xi=\xi(t)=\sigma(1, t)$, the contact shear, as an additional dependent variable.

The abstract weak form of problem (2.9)-(2.17) is: Find a triplet $\{u, \theta, \xi\}$ such that 


$$
\begin{aligned}
& \theta \in \mathscr{E}, \quad \theta^{\prime} \in \mathscr{E}^{\prime}, \quad u, u^{\prime} \in \mathscr{V}, \quad u^{\prime \prime} \in \mathscr{V}, \quad \xi \in L^{2}(0, T), \\
& u^{\prime \prime}+c^{2}\left(A u+d A u^{\prime}\right)+\gamma^{*} \xi=f, \\
& \theta^{\prime}+k B \theta+N \theta=g\left(\theta, \gamma u^{\prime}\right), \\
& \theta(0)=\theta_{0} \in H, \quad u(0)=u_{0} \in V, \quad u^{\prime}(0)=v_{0} \in H,
\end{aligned}
$$

and for all $w \in \mathscr{V}$,

$$
\int_{0}^{T} \xi \gamma w \mathrm{~d} t \leqslant \int_{0}^{T} \mu\left(\left|\gamma u^{\prime}-v_{*}\right|\right) h(\theta)\left(\left|\gamma u^{\prime}-v_{*}+\gamma w\right|-\left|\gamma u^{\prime}-v_{*}\right|\right) \mathrm{d} t .
$$

We have, under the above assumptions on the data,

\section{THEOREM 3.2. There exists a unique solution of problem (3.1)-(3.5).}

We prove the existence of a solution by considering a sequence of approximate problems obtained by retarding the arguments of $\theta$ and $\gamma u^{\prime}$ in $g$. We define the retardation operator $\tau_{\epsilon}$, for $\epsilon>0$, by

$$
\tau_{\epsilon} w(t) \equiv \begin{cases}w(t-\epsilon) & \text { if } t \geqslant \epsilon, \\ 0 & \text { if } t<\epsilon .\end{cases}
$$

Then, the approximate problem is: Find a triplet $\left\{u_{\epsilon}, \theta_{\epsilon}, \xi_{\epsilon}\right\}$ such that

$$
\begin{aligned}
& \theta_{\epsilon} \in \mathscr{E}, \quad \theta_{\epsilon}^{\prime} \in \mathscr{E}^{\prime}, \quad u_{\epsilon}, u_{\epsilon}^{\prime} \in \mathscr{V}, \quad u_{\epsilon}^{\prime \prime} \in \mathscr{V}^{\prime}, \quad \xi_{\epsilon} \in L^{2}(0, T), \\
& u_{\epsilon}^{\prime \prime}+c^{2}\left(A u_{\epsilon}+d A u_{\epsilon}^{\prime}\right)+\gamma^{*} \xi_{\epsilon}=f, \\
& \theta_{\epsilon}^{\prime}+k B \theta_{\epsilon}+N \theta_{\epsilon}=g\left(\tau_{\epsilon} \theta_{\epsilon}, \tau_{\epsilon} \gamma u_{\epsilon}^{\prime}\right), \\
& \theta_{\epsilon}(0)=\theta_{0} \in H, \quad u_{\epsilon}(0)=u_{0} \in V, \quad u_{\epsilon}^{\prime}(0)=v_{0} \in H,
\end{aligned}
$$

and for all $w \in \mathscr{V}$,

$$
\int_{0}^{T} \xi_{\epsilon} \gamma w \mathrm{~d} t \leqslant \int_{0}^{T} \mu\left(\left|\gamma u_{\epsilon}^{\prime}-v_{*}\right|\right) h\left(\theta_{\epsilon}\right)\left(\left|\gamma u_{\epsilon}^{\prime}-v_{*}+\gamma w\right|-\left|\gamma u_{\epsilon}^{\prime}-v_{*}\right|\right) \mathrm{d} t .
$$

It follows from the results in [2] and Lemma 3.1 that there exists a solution to (3.6)-(3.10) for each small $\epsilon>0$. Indeed, first the existence follows for $0 \leqslant t \leqslant \epsilon$, and then the solution is constructed step by step, for step length $\epsilon$, up to $t=T$.

We proceed to obtain a priori estimates on the approximate solutions. For the sake of simplicity we omit the subscript $\epsilon$, and use $C$ as a positive constant independent of $\epsilon$. We multiply (3.8) by $\theta$, integrate over $(0, t)$ and perform routine estimates to obtain

$$
\begin{aligned}
\left|\int_{0}^{t}\langle N(s, \theta(s)), \theta(s)\rangle \mathrm{d} s\right| & \leqslant \int_{0}^{t}\left(C+C\left(|\gamma \theta(s)|+\left|\theta_{t}(s)\right|\right)\right)|\gamma \theta(s)| \mathrm{d} s \\
& \leqslant C+C \int_{0}^{t}|\gamma \theta(s)|^{2} \mathrm{~d} s \leqslant C+C \int_{0}^{t}\|\theta(s)\|_{F}^{2} \mathrm{~d} s,
\end{aligned}
$$

where $F$ is an intermediate space such that $\gamma$ maps $F$ continuously into $\mathbb{R}$ and $E$ embeds compactly into $F$, e.g. $F=H^{3 / 4}(0,1)$. It follows from the compactness of the embedding that the term is majorized by

$$
C+C_{\delta} \int_{0}^{t}|\theta(s)|_{H}^{2} \mathrm{~d} s+\delta \int_{0}^{1}\|\theta(s)\|_{E}^{2} \mathrm{~d} s,
$$

where, by the definition of $E,\|\theta\|_{E}^{2} \equiv\left|\theta_{x}\right|_{H}^{2}$. Now, using the definition of $g$ and $\nu(r)=\mu(r) r$, we find 


$$
\begin{aligned}
\left|\int_{0}^{t}\left\langle g\left(\tau_{\epsilon} \theta, \tau_{\epsilon}\left(\gamma u^{\prime}\right)\right) \theta\right\rangle \mathrm{d} s\right| & \leqslant \int_{\epsilon}^{t} \nu\left(\left|\left(\gamma u^{\prime}\right)(s-\epsilon)-v_{*}(s)\right|\right) h(\theta(s-\epsilon)) \mid \gamma \theta(s) \mathrm{d} s \\
& =\int_{0}^{t-\epsilon} \nu\left(\left|\left(\gamma u^{\prime}\right)(s)-v_{*}(s+\epsilon)\right|\right) h(\theta(s))|\gamma \theta(s+\epsilon)| \mathrm{d} s \\
& \leqslant C \int_{0}^{1-\epsilon}\left(\left|\left(\gamma u^{\prime}\right)(s)\right|+C\right)|\gamma \theta(s+\epsilon)| \mathrm{d} s \\
& \leqslant C+C \int_{0}^{t}\left|\gamma u^{\prime}(s)\right|^{2} \mathrm{~d} s+C \int_{0}^{t}|\gamma \theta(s)|^{2} \mathrm{~d} s
\end{aligned}
$$

which is no larger than

$$
C+C_{\delta} \int_{0}^{t}\left|u^{\prime}(s)\right|_{H}^{2}+\delta \int_{0}^{t}\left\|u^{\prime}(s)\right\|_{V}^{2} \mathrm{~d} s+\delta \int_{0}^{t}\|\theta(s)\|_{E}^{2} \mathrm{~d} s+C_{\delta} \int_{0}^{t}|\theta(s)|_{H}^{2} \mathrm{~d} s .
$$

(3.11) and (3.12) imply that for $\delta$ small enough,

$$
\begin{aligned}
& \frac{1}{2}|\theta(t)|_{H}^{2}-\frac{1}{2}\left|\theta_{0}\right|_{H}^{2}+\frac{k}{2} \int_{0}^{t}\|\theta(s)\|_{E}^{2} \mathrm{~d} s \\
& \quad \leqslant C+C \int_{0}^{t}|\theta(s)|_{H}^{2} \mathrm{~d} s+C_{\delta} \int_{0}^{t}\left|u^{\prime}(s)\right|_{H}^{2}+\delta \int_{0}^{t}\left\|u^{\prime}(s)\right\|_{V}^{2} \mathrm{~d} s .
\end{aligned}
$$

An application of Gronwall's inequality yields

$$
|\theta(t)|_{H}^{2}+\int_{0}^{t}\|\theta(s)\|_{E}^{2} \mathrm{~d} s \leqslant C+C \int_{0}^{t}\left|u^{\prime}(s)\right|_{H}^{2}+C \int_{0}^{t}\left\|u^{\prime}(s)\right\|_{V}^{2} \mathrm{~d} s .
$$

Now, it follows from similar estimates on $u$ and (3.14) that

$$
|\theta(t)|_{H}^{2}+\int_{0}^{\prime}\|\theta(s)\|_{E}^{2} \mathrm{~d} s+\left|u^{\prime}(t)\right|_{H}^{2}+\|u(t)\|_{V}^{2}+\int_{0}^{\prime}\left\|u^{\prime}(s)\right\|_{V}^{2} \mathrm{~d} s \leqslant C .
$$

Also, it is straightforward to show that

$$
|\xi|_{L^{2}(0, T)} \leqslant|h|_{L^{2}(0, T)} \leqslant C .
$$

Therefore, there exists a subsequence $\left\{u_{\epsilon}, \theta_{\epsilon}, \xi_{\epsilon}\right\}$ of solutions to (3.6)-(3.10), for a sequence $\epsilon \rightarrow 0$, such that

$$
\begin{array}{ll}
u_{\epsilon}^{\prime} \rightarrow u^{\prime} & \text { weak* in } L^{\times}(0, T ; H), \\
u_{\epsilon}^{\prime \prime} \rightarrow u^{\prime \prime} & \text { weakly in } \mathscr{V}^{\prime \prime}, \\
u_{\epsilon} \rightarrow u & \text { strongly in } C(0, T ; W), \\
u_{\epsilon}^{\prime} \rightarrow u^{\prime} & \text { strongly in } L^{2}(0, T ; W), \\
u_{\epsilon}^{\prime}(t) \rightarrow u^{\prime}(t) & \text { in } W \text { a.e. } t, \\
u_{\epsilon} \rightarrow u & \text { weak* in } L^{\infty}(0, T ; V), \\
u_{\epsilon}^{\prime} \rightarrow u^{\prime} & \text { weakly in } \mathscr{V}, \\
\xi_{\epsilon} \rightarrow \xi & \text { weakly in } L^{2}(0, T), \\
\theta_{\epsilon}^{\prime} \rightarrow \theta^{\prime} & \text { weakly in } \mathscr{E}^{\prime}, \\
\theta_{\epsilon} \rightarrow \theta & \text { weakly in } \mathscr{E}, \\
\theta_{\epsilon} \rightarrow \theta & \text { strongly in } L^{2}(0, T ; F), \\
\theta_{\epsilon}(t) \rightarrow \theta(t) & \text { in } F \text { for a.e. } t \in[0, T] .
\end{array}
$$

Now, 


$$
\begin{aligned}
\left\langle g\left(\tau_{\epsilon} \theta_{\epsilon}, \tau_{\epsilon}\left(\gamma u_{\epsilon}^{\prime}\right)\right), \eta\right\rangle & =\int_{0}^{T} \mu\left(\left|\tau_{\epsilon}\left(\gamma u_{\epsilon}^{\prime}\right)-v_{*}(t)\right|\right)\left|\tau_{\epsilon}\left(\gamma u_{\epsilon}^{\prime}\right)-v_{*}(t)\right| h\left(\tau_{\epsilon} \theta_{\epsilon}\right) \gamma \eta \mathrm{d} t \\
& =\int_{\epsilon}^{T} \mu\left(\left|\tau_{\epsilon}\left(\gamma u^{\prime}\right)-v_{*}(t)\right|\right)\left|\tau_{\epsilon}\left(\gamma u^{\prime}\right)-v_{*}(t)\right| h\left(\tau_{\epsilon} \theta_{\epsilon}\right) \gamma \eta \mathrm{d} t+g(\epsilon),
\end{aligned}
$$

where,

$$
\begin{aligned}
g(\epsilon) & \leqslant \int_{0}^{T}\left|\nu\left(\left|\tau_{\epsilon}\left(\gamma u_{\epsilon}^{\prime}\right)-v_{*}(t)\right|\right)-\nu\left(\left|\tau_{\epsilon}\left(\gamma u^{\prime}\right)-v_{*}(t)\right|\right)\right| h\left(\tau_{\epsilon} \theta_{\epsilon}\right) \gamma \eta \mathrm{d} t \\
& \leqslant K \operatorname{Lip}_{\nu}\left(\int_{0}^{T}\left|\tau_{\epsilon}\left(\gamma u_{\epsilon}^{\prime}\right)-\tau_{\epsilon}\left(\gamma u^{\prime}\right)\right|\right)|\gamma \eta| \mathrm{d} t,
\end{aligned}
$$

where Lip ${ }_{v}$ is the Lipschitz constant of $\nu$, which converges to zero by (3.23).

The first term on the right-hand of (3.29) converges to

$$
\int_{0}^{T} \mu\left(\left|\gamma u^{\prime}-v_{*}(t)\right|\right)\left|\gamma u^{\prime}-v_{*}(t)\right| h(\theta) \gamma \eta \mathrm{d} t=\left\langle g\left(\theta, \gamma u^{\prime}\right), \eta\right\rangle,
$$

by Lemma 3.1, the continuity of translations in $L^{2}$ and the dominated convergence theorem. Therefore, the limits (3.17)-(3.28) are sufficient to pass to the limit in (3.6)-(3.10) and obtain (3.1)-(3.4). It remains to verify that $\xi$ satisfies (3.5). But, this follows from (3.10), the continuity of translations in $L^{2}$, the Lipschitz continuity of $h$ and the pointwise limits together with the dominated convergence theorem. This proves the existence part of the theorem.

PROOF OF UNIQUENESS. Suppose $\left\{u_{i}, \theta_{i}, \xi_{i}\right\}$, where here and below $i=1,2$, are two solutions to the problem. Then, for the mechanical part of the problem, we obtain

$$
\begin{gathered}
\frac{1}{2}\left|u_{1}^{\prime}(t)-u_{2}^{\prime}(t)\right|_{H}^{2}+\frac{c^{2}}{2}\left\|u_{1}(t)-u_{2}(t)\right\|_{V}^{2}+d \int_{0}^{t}\left\|u_{1}^{\prime}(s)-u_{2}^{\prime}(s)\right\|_{V}^{2} \mathrm{~d} s \\
=-\int_{0}^{t}\left(\xi_{1}(s)-\xi_{2}(s)\right)\left(\gamma u_{1}^{\prime}(s)-\gamma u_{2}^{\prime}(s)\right) \mathrm{d} s .
\end{gathered}
$$

We consider the last term in (3.30) and note that (3.10) implies

$$
\begin{aligned}
\int_{0}^{t} \xi_{2}(s)\left(\gamma u_{1}^{\prime}(s)-\gamma u_{2}^{\prime}(s)\right) \mathrm{d} s \leqslant & \int_{0}^{t} \mu\left(\left|\gamma u_{2}^{\prime}(s)-v_{*}(s)\right|\right) h\left(\theta_{2}\right)(s) \\
& \times\left(\left|\gamma u_{2}^{\prime}(s)-v_{*}(s)+\gamma u_{1}^{\prime}(s)-\gamma u_{2}^{\prime}(s)\right|-\left|\gamma u_{2}^{\prime}(s)-v_{*}(s)\right|\right) \mathrm{d} s,
\end{aligned}
$$

and

$$
\begin{aligned}
- & \int_{0}^{t} \xi_{1}(s)\left(\gamma u_{1}^{\prime}(s)-\gamma u_{2}^{\prime}(s)\right) \mathrm{d} s \\
= & \int_{0}^{1} \xi_{1}(s)\left(\gamma u_{2}^{\prime}(s)-\gamma u_{1}^{\prime}(s)\right) \mathrm{d} s \leqslant \int_{0}^{1} \mu\left(\left|\gamma u_{1}^{\prime}(s)-v_{*}(s)\right|\right) h\left(\theta_{1}\right)(s) \\
& \times\left(\left|\gamma u_{1}^{\prime}(s)-v_{*}(s)+\gamma u_{2}^{\prime}(s)-\gamma u_{1}^{\prime}(s)\right|-\left|\gamma u_{1}^{\prime}(s)-v_{*}(s)\right|\right) \mathrm{d} s .
\end{aligned}
$$

Therefore, the last term in (3.30) is bounded by

$$
\begin{aligned}
& \int_{0}^{\prime}\left(\mu\left(\left|\gamma u_{1}^{\prime}(s)-v_{*}(s)\right|\right) h\left(\theta_{1}\right)(s)-\mu\left(\left|\gamma u_{2}^{\prime}(s)-v_{*}(s)\right|\right) h\left(\theta_{2}\right)(s)\right) \\
& \quad \times\left(\left|\gamma u_{2}^{\prime}(s)-v_{*}(s)\right|-\left|\gamma u_{1}^{\prime}(s)-v_{*}(s)\right|\right) \mathrm{d} s \\
& \quad \leqslant \int_{0}^{\prime}\left(\mu\left(\left|\gamma u_{1}^{\prime}(s)-v_{*}(s)\right|\right)-\mu\left(\left|\gamma u_{2}^{\prime}(s)-v_{*}(s)\right|\right)\left|h\left(\theta_{1}\right)(s)\right| \gamma u_{2}^{\prime}(s)-\gamma u_{1}^{\prime}(s) \mid \mathrm{d} s\right.
\end{aligned}
$$




$$
+\mu_{0} \int_{0}^{\prime}\left|h\left(\theta_{2}\right)(s)-h\left(\theta_{1}\right)(s)\right|\left|\gamma u_{2}^{\prime}(s)-\gamma u_{1}^{\prime}(s)\right| \mathrm{d} s,
$$

which, by Lemma 3.1, is bounded by

$$
\begin{aligned}
& C \int_{0}^{1}\left|\gamma u_{2}^{\prime}(s)-\gamma u_{1}^{\prime}(s)\right|^{2} \mathrm{~d} s+C \int_{0}^{t}\left|\theta_{1}(s)-\theta_{2}(s)\right|_{H}\left|\gamma u_{2}^{\prime}(s)-\gamma u_{1}^{\prime}(s)\right| \mathrm{d} s \\
& \quad \leqslant C \int_{0}^{1}\left|\gamma u_{2}^{\prime}(s)-\gamma u_{1}^{\prime}(s)\right|^{2} \mathrm{~d} s+C \int_{0}^{t}\left|\theta_{1}(s)-\theta_{2}(s)\right|_{H}^{2} \mathrm{~d} s,
\end{aligned}
$$

which in turn is bounded, by the compactness of the embedding of $V$ into a space on which the trace map is continuous, by

$$
\leqslant \delta \int_{0}^{t}\left\|u_{2}^{\prime}-u_{1}^{\prime}\right\|_{V}^{2} \mathrm{~d} s+C_{\delta} \int_{0}^{t}\left|u_{2}^{\prime}-u_{1}^{\prime}\right|_{H}^{2} \mathrm{~d} s+C \int_{0}^{\prime}\left|\theta_{1}-\theta_{2}\right|_{H}^{2} \mathrm{~d} s
$$

Choosing $\delta$ small enough (3.30) yields

$$
\begin{gathered}
\left|u_{1}^{\prime}(t)-u_{2}^{\prime}(t)\right|_{H}^{2}+\left\|u_{1}(t)-u_{2}(t)\right\|_{V}^{2}+C d \int_{0}^{t}\left\|u_{1}^{\prime}(s)-u_{2}^{\prime}(s)\right\|_{V}^{2} \mathrm{~d} s \\
\leqslant C_{\delta} \int_{0}^{t}\left|u_{2}^{\prime}(s)-u_{1}^{\prime}(s)\right|_{H}^{2} \mathrm{~d} s+C \int_{0}^{t}\left|\theta_{1}(s)-\theta_{2}(s)\right|_{H}^{2} \mathrm{~d} s .
\end{gathered}
$$

Now, Gronwall's inequality implies

$$
\begin{aligned}
& \left|u_{1}^{\prime}(t)-u_{2}^{\prime}(t)\right|_{H}^{2}+\left\|u_{1}(t)-u_{2}(t)\right\|_{V}^{2}+C d \int_{0}^{\prime}\left\|u_{1}^{\prime}(s)-u_{2}^{\prime}(s)\right\|_{V}^{2} \mathrm{~d} s \\
& \quad \leqslant C \int_{0}^{t}\left|\theta_{1}(s)-\theta_{2}(s)\right|_{H}^{2} \mathrm{~d} s .
\end{aligned}
$$

Letting $F=H^{3 / 4}(0,1)$, it follows that the trace map $\gamma$ maps $F$ continuously into $\mathbb{R}$. Therefore, we have

$$
|\gamma \eta| \leqslant \epsilon\|\eta\|_{E}+C_{\epsilon}|\eta|_{H}, \quad|\gamma w| \leqslant \epsilon\|\boldsymbol{w}\|_{V}+C_{\epsilon}|w|_{H} .
$$

Since $h_{h}$ is Lipschitz,

$$
\begin{aligned}
\left|\left\langle N\left(t, \theta_{1}\right)-N\left(t, \theta_{2}\right), \theta_{1}-\theta_{2}\right\rangle\right| & \leqslant C\left|\gamma \theta_{1}-\gamma \theta_{2}\right|^{2} \\
& \leqslant \epsilon\left\|\theta_{1}-\theta_{2}\right\|_{E}^{2}+C_{\epsilon}\left|\theta_{1}-\theta_{2}\right|_{H}^{2} .
\end{aligned}
$$

Now, we consider $g$, and find

$$
\left\langle g\left(\theta_{1}, \gamma u_{1}^{\prime}\right)-g\left(\theta_{2}, \gamma u_{2}^{\prime}\right), \theta_{1}-\theta_{2}\right\rangle=\left(\nu\left(\left|\gamma u_{1}^{\prime}-v_{*}\right|\right) h\left(\theta_{1}\right)-\nu\left(\left|\gamma u_{2}^{\prime}-v_{*}\right|\right) h\left(\theta_{2}\right)\right)\left(\gamma \theta_{1}-\gamma \theta_{2}\right) .
$$

Lemma 3.1 and the assumption that $v_{*}$ is bounded imply

$$
\begin{aligned}
&\left|\left\langle g\left(\theta_{1}, \gamma u_{1}^{\prime}\right)-g\left(\theta_{2}, \gamma u_{2}^{\prime}\right), \theta_{1}-\theta_{2}\right\rangle\right| \leqslant C\left\{\left|\gamma u_{1}^{\prime}-\gamma u_{2}^{\prime}\right|\left|\gamma \theta_{1}-\gamma \theta_{2}\right|\right. \\
&\left.\quad+\left|v\left(\left|\gamma u_{2}^{\prime}-v_{*}\right|\right)\right|\left|\theta_{1}-\theta_{2}\right|_{H}\left|\gamma \theta_{1}-\gamma \theta_{2}\right|\right\} \\
& \leqslant C\left\{\left|\gamma u_{1}^{\prime}-\gamma u_{2}^{\prime}\right|^{2}+\epsilon\left\|\theta_{1}-\theta_{2}\right\|_{E}^{2}+C_{\epsilon}\left|\theta_{1}-\theta_{2}\right|_{H}^{2}\right. \\
&\left.+\left(1+\left\|u_{2}^{\prime}\right\|_{V}^{2}\right)\left|\theta_{1}-\theta_{2}\right|_{H}^{2}+\epsilon\left\|\theta_{1}-\theta_{2}\right\|_{E}^{2}+C_{\epsilon}\left|\theta_{1}-\theta_{2}\right|_{H}^{2}\right\} .
\end{aligned}
$$

Therefore, when $\epsilon$ is small enough,

$$
\left|\theta_{1}(t)-\theta_{2}(t)\right|_{H}^{2}+\int_{0}^{t}\left\|\theta_{1}-\theta_{2}\right\|_{E}^{2} \mathrm{~d} s \leqslant C \int_{0}^{t}\left|\gamma u_{1}^{\prime}-\gamma u_{2}^{\prime}\right|^{2} \mathrm{~d} s+\int_{0}^{t}\left(C_{\epsilon}+\left\|u_{2}^{\prime}\right\|_{V}^{2}\right)\left|\theta_{1}-\theta_{2}\right|_{H}^{2} \mathrm{~d} s .
$$

Gronwall's inequality now yields 


$$
\left|\theta_{1}(t)-\theta_{2}(t)\right|_{H}^{2} \leqslant C \exp \left(\int_{0}^{T}\left(C_{\epsilon}+|| u_{2}^{\prime} \|_{V}^{2}\right) \mathrm{d} s\right) \int_{0}^{\prime}\left|\gamma u_{1}^{\prime}-\gamma u_{2}^{\prime}\right|^{2} \mathrm{~d} s \leqslant C_{\epsilon} \int_{0}^{t}\left|\gamma u_{1}^{\prime}-\gamma u_{2}^{\prime}\right|^{2} \mathrm{~d} s .
$$

Using this estimate in (3.31) we obtain

$$
\begin{aligned}
& \left|u_{1}^{\prime}(t)-u_{2}^{\prime}(t)\right|_{H}^{2}+\left\|u_{1}(t)-u_{2}(t)\right\|_{V}^{2}+C d \int_{0}^{t}\left\|u_{1}^{\prime}(s)-u_{2}^{\prime}(s)\right\|_{V}^{2} \mathrm{~d} s \\
& \quad \leqslant C_{\epsilon} \int_{0}^{t} \int_{0}^{s}\left|\gamma u_{1}^{\prime}-\gamma u_{2}^{\prime}\right|^{2} \mathrm{~d} r \mathrm{~d} s \leqslant C_{\epsilon} \int_{0}^{t} \int_{0}^{s}\left\|u_{1}^{\prime}-u_{2}^{\prime}\right\|_{V}^{2} \mathrm{~d} r \mathrm{~d} s .
\end{aligned}
$$

Using Gronwall's inequality again shows that

$$
0=\int_{0}^{t}\left\|u_{1}^{\prime}-u_{2}^{\prime}\right\|_{V}^{2} \mathrm{~d} s
$$

and, thus, $u_{1}=u_{2}$. It follows now from (3.33) that $\theta_{1}=\theta_{2}$. Finally, we have $\xi_{1}=\xi_{2}$, since by (3.5) $\int_{0}^{T}\left(\xi_{1}-\xi_{2}\right) \gamma w \mathrm{~d} t \leqslant 0$, for all $w \in \mathscr{V}$. This proves the theorem.

\section{Numerical algorithms and simulations}

We describe an algorithm for the problem and present numerical simulations of the solutions. The numerical algorithm is based on a Crank-Nicholson finite differences scheme. For the sake of simplicity we consider the elastic case, when $d=0$, and when there are no applied forces, $f=0$.

We denote by $V$ the column vector $\left(u_{t}(x, t), u_{x x}(x, t), \theta(x, t)\right)$, then the evolution equations can be written as

$$
\frac{\partial}{\partial t} V=\frac{\partial^{2}}{\partial x^{2}}(D V)
$$

with

$$
D=\left(\begin{array}{ccc}
0 & -c^{2} & 0 \\
1 & 0 & 0 \\
0 & 0 & k
\end{array}\right)
$$

Let $V^{j, n}=\left(u_{t}^{j, n}, u_{x x}^{j, n}, \theta^{j, n}\right)$ approximate $V$ at time $t^{n}=n \Delta t$ and position $x_{j}=j \Delta x\left(j=0, \ldots, N_{x}\right)$, the CrankNicholson scheme reads as follows:

$$
\frac{1}{\Delta t}\left(V^{j, n+1}-V^{j, n}\right)=\frac{D}{2(\Delta x)^{2}}\left(V^{j+1, n+1}-2 V^{j, n+1}+V^{j-1 . n+1}+V^{j+1 . n}-2 V^{j, n}+V^{j-1, n}\right) .
$$

An implicit scheme was chosen since such a scheme is stable, and permits to avoid numerical instabilities (see e.g. [4]) due to the friction condition, which may be considered as a stiffness condition (in fact nondifferentiable and multivalued).

The boundary conditions are of two kinds. The conditions $u(0, t)=0, \theta(0, t)=\theta_{l}$ and $u_{x x}(1, t)=0$, can be approximated as

$$
u_{i}^{0 . n}=0, \quad \theta^{0, n}=\theta_{l}^{n}, \quad u_{x x}^{N_{x} \cdot n}=0 .
$$

We use the virtual point method to discretize the thermal boundary condition and the condition $u_{x}(0, t)=0$. Indeed, for the condition $u_{x}(0, t)=0$, we introduce the virtual point $u^{-1, n}$, and we have

$$
u_{t}(0+h, t)=u_{x, x}(0, t) h^{2}+o\left(h^{2}\right),
$$

if $u$ is sufficiently smooth. Thus, for an order two approximation we use $u^{-1, n}=u^{1, n}$. Applying now the Crank-Nicholson scheme yields

$$
u_{x x}^{0, n+1}-\frac{\Delta t}{(\Delta x)^{2}} u_{t}^{1, n+1}=u_{x x}^{0, n}+\frac{\Delta t}{(\Delta x)^{2}} u_{t}^{1, n} .
$$


We apply the same method to the thermal boundary condition at the contact point, and this leads to a nonlinear equation.

The friction condition can be divided into two: the stick and the slip conditions, which are of different type. This leads to two nonlinear systems: one for the solution with the stick condition and the other one for the slip solution. When the solution of the continuous model is unique, we expect that at each time step only one of these two holds true. The stick solution is valid if $|\sigma(1, t)|<\mu h(t)$, and the slip solution holds when $|\sigma(1, t)|=\mu h(t)$.

To solve the system, which contains two coupled nonlinear equations corresponding to the friction and thermal conditions, we apply a fixed point method. At each time step we make a first guess for the contact pressure, the friction force and the value of $\theta_{x}^{N_{x}{ }^{n+1}}$ by taking the values from the previous time step. Then, we solve two linear systems and compute the corresponding values of the contact pressure, friction force and $\theta_{x}^{N_{x}, n+1}$, and then we iterate by repeating the process.

\subsection{Numerical simulations}

We now describe a number of numerical simulations of the problem. The purpose of these simulations is to show different types of behavior that the model solutions exhibit. We present only four simulations, a more thorough investigation will be performed in the sequel. Our main interest lies in the stick-slip phenomenon, and the values of the coefficients were chosen accordingly, and not necessarily for a specific material or setting.

For each simulation we show the sliding velocity, the temperature and the Fourier transform of the velocity at
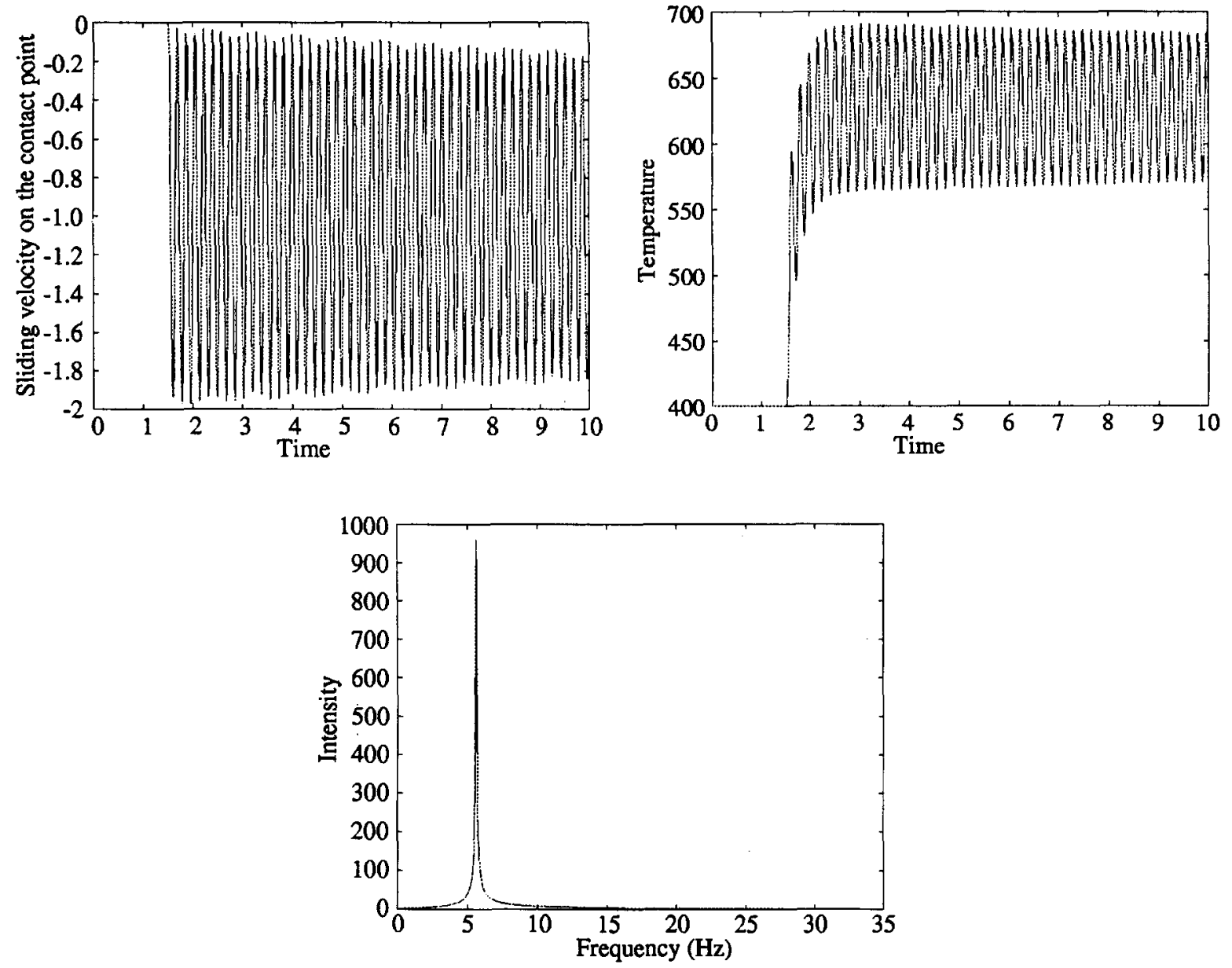

Fig. 2. The case with $\mu=$ const. and $v_{*}=$ const. 
the contact point. The last is meant to indicate the possible noise that the slip/stick motion of the contacting end produces. The constants are chosen as $c=10, k=1, h_{b}=1, \alpha=0.01, \beta=0$ and $\theta_{l}=\theta_{b}=400$.

The first simulation, presented in Fig. 2, is a simple case where the friction coefficient is constant, $\mu=0.9$, and the velocity of the foundation is also constant $v_{*}=1$. The initial condition is $u_{0}=0$. In the first period, the beam remains stuck to the surface and the system is under loading. The beam begins to slide when the shear stress threshold, given in the friction law, is reached. Then, the solution is oscillatory about the stationary solution, with a slow convergence toward the stationary solution.

The end oscillations are large, and so are the temperature oscillations, both due to the relatively large coefficient of friction. There is a sharp peak in the frequency domain near $6 \mathrm{~Hz}$, the natural first frequency of this beam.

In the second simulation, presented in Fig. 3, the rigid foundation oscillates with velocity

$$
v_{*}(t)=1+2 \sin \left(\frac{2 \pi}{T} t\right) .
$$

In this particular case, the frequency is equal to $3 \mathrm{~Hz}(T=1 / 3)$. This leads to a stick-slip phenomenon. Indeed, the end undergoes rapid oscillations with periods of stick, where it moves with the obstacle. The frequency distribution is richer, and more interesting. Higher modes are excited, but the three main peaks are below $10 \mathrm{~Hz}$, the smaller ones above it.

The third simulation, shown in Fig. 4, deals with the case of slip dependent coefficient of friction and constant velocity of the rigid foundation $v_{*}=1$. The slip dependence of $\mu$ is shown in the fourth graph of Fig. 4. A
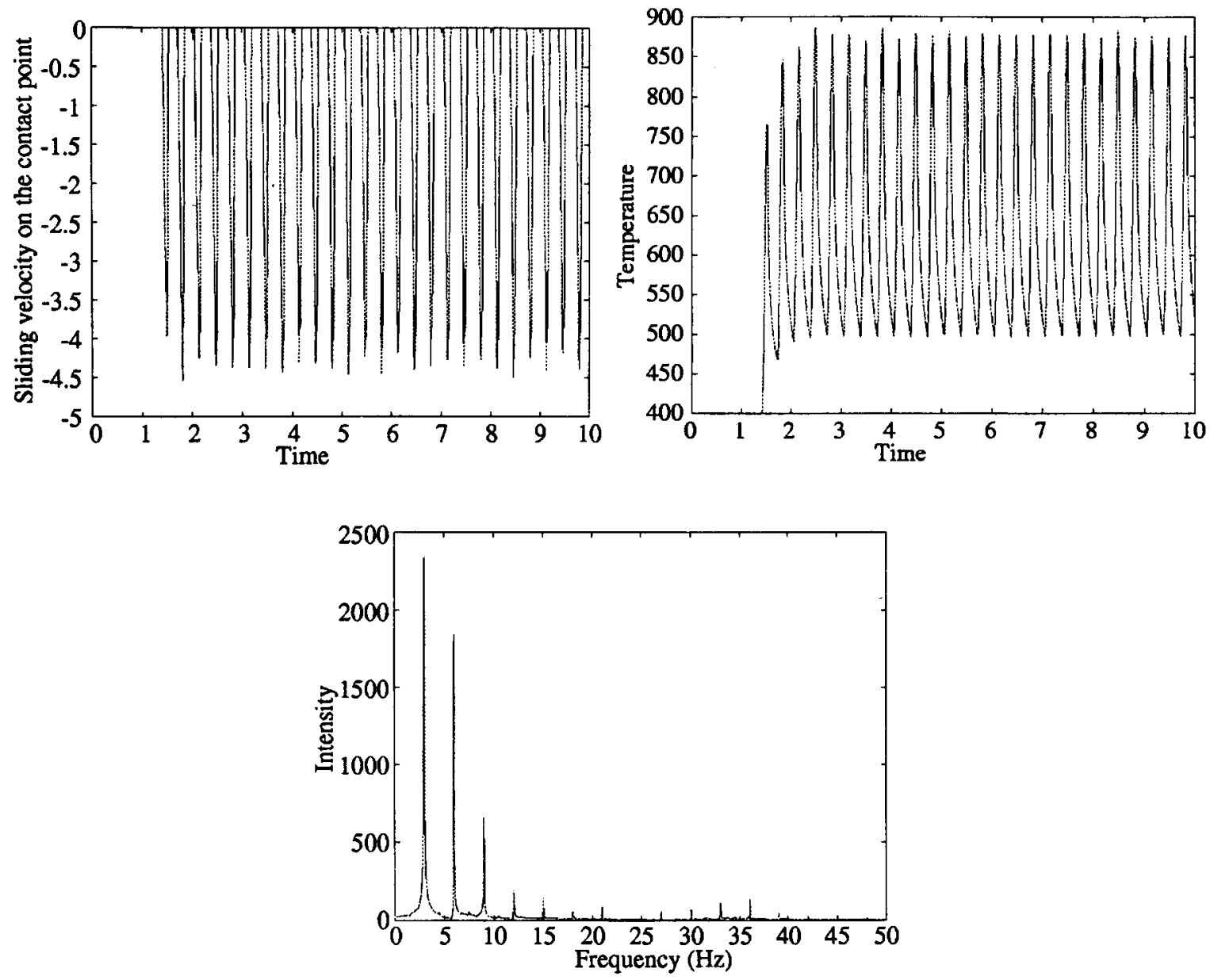

Fig. 3. The case with periodically oscillating $v_{*}$. 

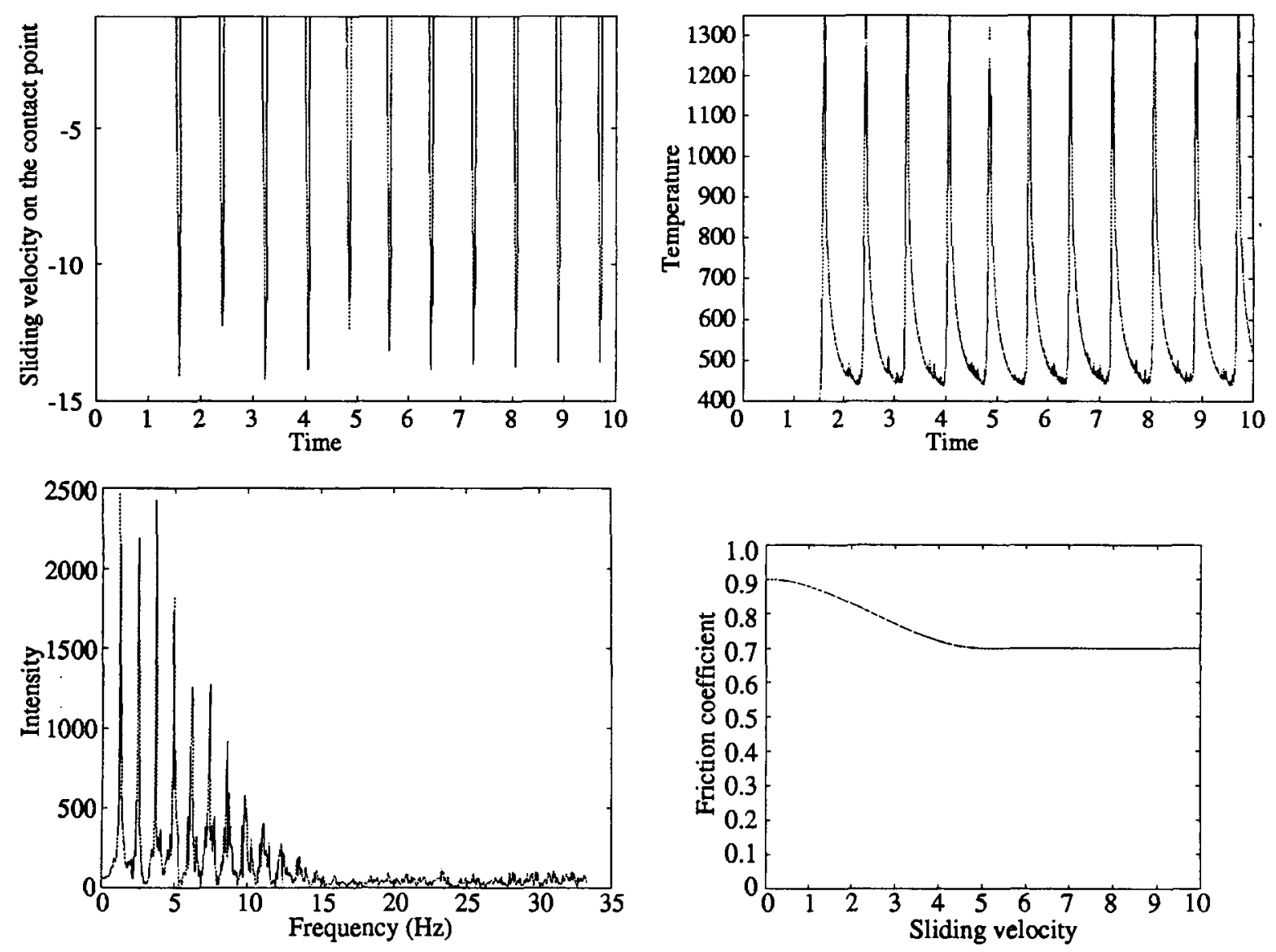

Fig. 4. The coefficient of friction is decreasing and $v_{*}=1$.

decreasing coefficient of this form is often used to model the stick-slip behavior (see e.g. [8] or [9]), and we observe that this leads to this kind of behavior in the present model, too. Indeed, even a gently decreasing $\mu$ leads to a fairly 'noisy' behavior, which usually is assumed to be caused by a discontinuous coefficient of friction, with different static and dynamic values. The oscillations of the end and of the temperature are noticeable.

In the final example, depicted in Fig. 5, the friction coefficient is chosen to depend on the temperature at the contact point (the dependence is shown in the fourth graph of Fig. 5). The temperature leads to two opposite effects: reduction of the friction force due to the decrease in the friction coefficient and the beam's thermal expansion which increases the contact pressure, and thus the friction force. In the example presented in Fig. 5, there is a stick-slip behavior similar to the one with a slip velocity dependent friction coefficient, but with a lower frequency of the oscillations.

Clearly, even the 'simple' one-dimensional system exhibits varied and interesting types of behavior.

\section{Steady states}

We investigate the steady solutions to problem (2.9)-(2.17), with variable $\mu$. But we use the usual statement of Coulomb's law where $h=p$ and the friction bound is $\mu p$ (i.e. we set $\beta=0$ ). We find that when the heat generation is too large, the system does not have a steady state.

We note that in the above setting, only slip can take place in the steady state, which leads to the simplification of conditions (2.17). It follows from the model (2.9)-(2.17), when all the functions are time independent, that the steady problem is: Find a pair $\{\bar{\theta}=\bar{\theta}(x), \bar{u}=\bar{u}(x)\}$ such that 

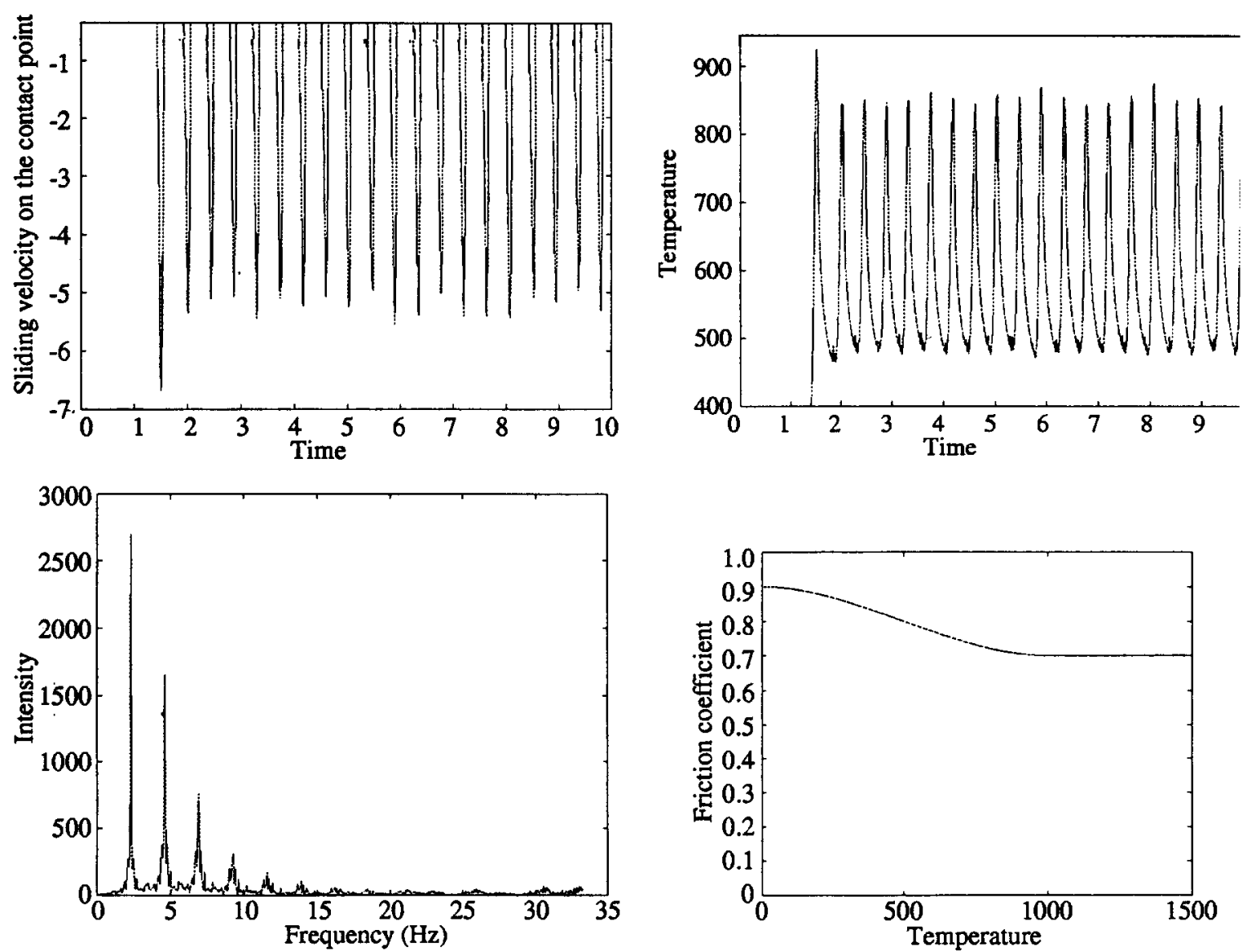

Fig. 5. The case with temperature dependent $\mu$.

$$
\begin{aligned}
& \bar{u}^{\prime \prime \prime \prime}=0, \quad \bar{\theta}^{\prime \prime}=0 \quad x \in(0,1), \\
& \bar{u}(0)=0, \quad \bar{u}^{\prime}(0)=0, \quad \bar{\theta}(0)=0, \\
& \bar{u}^{\prime \prime}(1)=0, \\
& k \bar{\theta}^{\prime}(1)=\mu\left(v_{*}\right) v_{*} \alpha \int_{0}^{1} \bar{\theta}(x) \mathrm{d} x-h_{b}\left(\bar{\theta}(1)-\theta_{b}\right), \\
& \left|\bar{u}^{\prime \prime \prime}(1)\right|=\mu\left(v_{*}\right) \alpha \int_{0}^{1} \bar{\theta}(x) \mathrm{d} x .
\end{aligned}
$$

Here, $v_{*}$ is the constant sliding velocity, for convenience chosen in the upward (positive) direction, $p=$ const., and for the sake of simplicity we set $c=1, \theta_{t}=0$ and $f=0$. Also, we recall that $p=\alpha \int_{0}^{1} \bar{\theta}(x) \mathrm{d} x$.

A straightforward calculation shows that the unique solution of the steady state problem is

$$
\begin{aligned}
& \bar{u}(x)=\frac{1}{4} \alpha \mu\left(v_{*}\right) A x^{2}\left(1-\frac{1}{3} x\right), \\
& \bar{\theta}(x)=A x,
\end{aligned}
$$

where

$$
A=\frac{h_{b} \theta_{b}}{h_{b}+k-\frac{1}{2} \alpha \mu\left(v_{*}\right) v_{*}},
$$

and the contact pressure is $p=\frac{1}{2} \alpha A$. 
This solution is valid only when the frictional heat generation is not too large. Indeed, assume first that $\nu(r)=r \mu(r)$ is monotone increasing, and let $v_{* *}$ be the solution of

$$
h_{b}+k=\frac{1}{2} \alpha \mu\left(v_{* *}\right) v_{* *},
$$

then, for all $v_{*}<v_{* *}$ the solution (5.6) and (5.7) holds. But we note that $A \rightarrow \infty$ as $v_{*} \rightarrow v_{* *}$. If $\nu(r)$ is monotone decreasing then the steady state exists for $v_{*}>v_{* *}$.

Finally, if $\nu(r)$ is neither, there may be intervals of $v_{*}$ for which the steady solution exists. While intuitively obvious, these results are of considerable applied interest since they determine the long time behavior of the solutions.

The stability properties of the steady states, when existing, are an open question, and so is the rate of convergence of the solution to the steady state.

\section{Conclusions}

We presented a 'simple' one-dimensional model for the thermomechanical behavior of a beam that is in frictional contact with a rigid moving obstacle. The problem is very interesting, and the numerical simulations indicate that the system exhibits different types of behavior. Our main interest was in the stick-slip phenomenon, which can be seen for slip dependent coefficient of friction, but the dependence was rather weak, while in many engineering investigations the coefficient of friction is assumed to jump from a static value to a smaller dynamic value. We plan to investigate such problems, but mathematically they are harder and pose more challenge. Moreover, the temperature oscillations indicate that the thermal effects must be included in any consideration of such processes; when the heat generation is too large, there are no steady states and the system is thermally unstable.

This research is a step in our investigation into the vibration and noise characteristics of systems in frictional contact. Clearly, we have only touched the tip of the iceberg.

\section{References}

[1] A. Amassad, M. Shillor and M. Sofonea, A quasistatic contact problem with slip dependent coefficient of friction, Math. Meth. Appl. Sci.. to appear.

[2] K.T. Andrews, M. Shillor and S. Wright, On the dynamic vibrations of an elastic beam in frictional contact with a rigid obstacle, J. Elasticity 42 (1996) $1-30$.

[3] J. Barber, J. Dundurs and M. Comninou, Stability considerations in thermoelastic contact. J. Appl. Mech. 47 (1980) $871-874$

141 A. Dontchev and F. Lempio. Difference methods for differential inclusions: a survey, SIAM Rev. 378 (1992).

[5] I.R. Ionescu and J.-C. Paumier, On the contact problem with slip dependent friction in elastodynamics, Europ. J. Mech. A/Solids 13(4) ( 1994$)$ 555-568.

[6] K.L. Kuttler. Time dependent implicit evolution equations, Nonlinear Anal. 10(5) (1986) 447-463.

[7] K.L. Kuttler and M. Shillor, Set-valued pseudomonotone maps and degenerate evolution inclusions, Comm. Contemp. Math., to appear.

[8] E. Rabinowicz, The intrinsic variables affecting the stick-slip process, Proc. Roy. Phys. Soc. 71 (1958) $668-675$.

[9] Y. Renard, Singular perturbation approach to an elastic dry friction problem with a non-monotone coefficient, Quart. Appl. Math., to appear.

[10] N. Strömberg, L. Johansson and A. Klarbring, Derivation and analysis of a generalized standard model for contact friction and wear, Int. J. Solids Struct. 13 (1996) 1817-1836.

[11] J. Telega, Topics on unilateral contact problems of elasticity and inelasticity, Preprint.

[12] T. Yeo and J. Barber, Finite element analysis of thermoelastic contact stability, J. Appl. Mech. 61 (1994) 919-922. 